\section{B) O Observatori de \\ Bioètica i Dret}

ISSN: $1886-5887$

\section{Revista de Bioética y Derecho}

Publicación del Máster en Bioética y Derecho nww.bioeticayderecho.ub.edu

DESDE EL MÁSTER

\title{
Problemas legales de los sistemas de notificación de incidentes. ¿Qué opinan los profesionales sanitarios?
}

\section{Legal problems of incident reporting systems. What is the opinion of health professionals?}

\author{
Blanca Obón Azuara * \\ ISABEl GutiÉRREZ Cía *
}

En esta sección tienen cabida las aportaciones de los alumnos del Máster en Bioética y Derecho de la Universitat de Barcelona (www.bioeticayderecho.ub.edu/master), de la presente edición (XVI Promoción 2013-2014) así como de ediciones anteriores. Esperamos vuestras contribuciones en obd2@ub.edu.

* Blanca Obón Azuara. Doctora en Medicina. Especialista en Medicina Intensiva. Servicio de Medicina Preventiva. Hospital Clínico Universitario de Zaragoza. blankaobona@hotmail.com

* Isabel Gutiérrez Cía. Doctora en Medicina. Especialista en Medicina Intensiva. Servicio de Medicina Intensiva. Hospital Clínico Universitario de Zaragoza. gutierrecilla@gmail.com 


\section{Índice}

Objetivo

Método

Resultados

Conclusiones

Bibliografía

\section{Resumen}

Objetivo: averiguar la percepción que los profesionales sanitarios tienen sobre los problemas legales de los sistemas de notificación de incidentes (SNI).

Método: estudio transversal realizado en la Comunidad Autónoma de Aragón, mediante una encuesta realizada "ad hoc"

Resultados: El 47,2\% considera que el paciente tiene derecho a conocer la seguridad del Sistema Sanitario, un $76,4 \%$ opina que el paciente tiene derecho a conocer si ha existido algún problema de seguridad durante su atención. El 77\% está de acuerdo con investigar los incidentes, incluso cuando de esta investigación pueda derivarse una sanción penal o administrativa..El 67,4\% cree que se pueden derivar consecuencias legales de la notificación, el 50\% desconoce si el declarar confiere protección al declarante. El 63\% considera que la información que contienen los SNI puede ser empleada en un juicio, un $42,4 \%$ considera que este tipo de sistemas puede constituir un modo de delación.

Conclusiones: La notificación de incidentes es una necesidad sentida por todos los profesionales, aunque ello suponga el riesgo de una sanción penal. Los profesionales desconocen las implicaciones legales de los SNI.

Palabras clave: sistemas de notificación de incidentes; seguridad del paciente; opinión de los profesionales; cuestiones legales. 


\section{Abstract}

Objective: To study the perception that health professionals have on legal problems of incident reporting systems (IRS).

Method: cross-sectional study in Aragon, we use a survey created for this purpose.

Results: 123 profesionals answer the survey; $47.2 \%$ believed that the patient has the right to be informed about the safety of the National Health System. $76.4 \%$ believed the patient has the right to receive information about their adverse events. $77 \%$ agreed about investigating the incidents, even when this research could imply a legal sanction. $67.4 \%$ believed that notification could have legal consequences; $50 \%$ did not know if the Law provides protection the deponent; $63 \%$ thougth the information registered in the IRS can be used in a lawsuit, $42.4 \%$ believed that such systems could be some way of delation.

Conclusions: Health professionals believe that IRS are useful, though they could be a source of legal problems. Health professionals do not know the legal problems of IRS.

Key words: reporting systems; patient safety; survey; legislation. 


\section{Objetivo}

La seguridad del paciente (SP) implica la práctica de una atención a la salud exenta de daños evitables, lo que supone, por una parte desarrollar sistemas y procesos dirigidos a reducir la probabilidad de aparición de fallos del sistema y errores de las personas, aumentando la probabilidad de detectarlos cuando suceden y mitigar sus consecuencias ${ }^{1,2}$.

El procurar beneficios a las personas y no originar daños evitables o innecesarios constituye uno de los deberes éticos de la medicina. Ambos principios, el de beneficencia y el de no maleficencia se aúnan en el clásico "Primun non nocere"3. Así, resulta evidente que los principios éticos que rigen la práctica de la Medicina se imbrican íntimamente en la atención segura al paciente. Beneficencia, no maleficencia, justicia y autonomía del paciente hablan el lenguaje de la seguridad ${ }^{4}$.

Resulta obvio en los dos primeros, la asistencia segura implica necesariamente evitar el daño y procurar el bien del paciente. Además en virtud del principio de autonomía del paciente, los profesionales sanitarios deben considerar la voluntad del mismo y promover su implicación en la toma de decisiones que puedan afectar a su salud. La comunicación del riesgo asistencial es una labor que forma parte del trabajo de los profesionales sanitarios ${ }^{5}$.

Con el principio de autonomía se alinea el derecho del paciente, universalmente admitido, de ser informado de los incidentes que le han causado daño ${ }^{6}$. Sin embargo en muchas ocasiones no se respeta este derecho y lo publicado hasta ahora sugiere que al menos un tercio de los pacientes no son informados incluso cuando se trata de graves eventos adversos (EA). Y entre aquellos que sí lo son se desconoce el alcance y la claridad de la información que reciben. Esta falta de información ha fomentado la creencia entre los pacientes de que los médicos ocultan sus errores ${ }^{7}$.

Los estudios epidemiológicos realizados hasta ahora $^{8}$ revelan que en ocasiones, las actuaciones médicas provocan algún tipo de perjuicio o daño al enfermo. Si además existe un deterioro o una no cuidada relación médico-paciente, es más que probable que surjan las reclamaciones y demandas ${ }^{9}$. En este sentido organizaciones y profesionales tienen la obligación de reparar el daño causado en virtud del principio de beneficencia ${ }^{10}$.

No sólo los profesionales, de forma individual, son responsables de la SP. Las organizaciones tienen hacia sus usuarios las mismas obligaciones éticas. En este sentido cobra especial relevancia el principio de justicia, ya que los costes de la no seguridad, con su elevado impacto económico y social, repercuten sobre la globalidad de los usuarios del sistema sanitario ${ }^{11}$.

En este sentido, en el año 2004 la Organización Mundial de la Salud (OMS) reconoció que la seguridad del paciente es un problema mundial. Nació así la Alianza Mundial por la Seguridad del Paciente, creada con el propósito de coordinar, difundir y acelerar las mejoras en materia de SP en todo el mundo ${ }^{12}$.

En nuestro país el Ministerio de Sanidad y Política Social e igualdad (MSPSI), en su responsabilidad de mejorar la calidad del sistema sanitario en su conjunto, como establece la Ley 
16/2003, de Cohesión y Calidad del Sistema Nacional de Salud, ha situado la seguridad del paciente en el centro de las políticas sanitarias como uno de los elementos clave de la mejora de la calidad, quedando así reflejado en la estrategia número 8 del Plan de Calidad para el Sistema Nacional de Salud (SNS). Y es en este contexto dónde se plantea la necesidad de diseñar y establece sistemas de información y notificación de EA para el aprendizaje ${ }^{13}$.

El desarrollo de sistemas de notificación de incidentes (SNI) surge inicialmente con el propósito de intentar disminuir la probabilidad de su aparición a través del aprendizaje basado en el análisis de los errores y el de los factores implicados.

El paciente que sufre un EA se encuentra en una situación de vulnerabilidad que exige la máxima delicadeza y consideración. Sin embargo se debe considerar que no es la única víctima del incidente. El profesional sanitario resulta habitualmente afectado por los EA en los que se ve implicado y con frecuencia experimenta sentimientos muy similares a los del paciente, angustia, ansiedad, incluso stress post traumático ${ }^{14,15}$. El profesional es consciente de que, por el principio de beneficencia, la reparación del daño no debe ser sólo económica sino también emocional lo que implica la necesidad de pedir perdón cuando existe evidencia de error, ya sea de un profesional o del sistema ${ }^{16}$. Del mismo modo el principio no maleficencia hace necesaria la notificación y el análisis del incidente, para evitar que vuelva a repetirse ${ }^{10}$.

Resulta fácil comprender la dualidad a la que se enfrenta el profesional que se encuentra implicado en un EA. Por una parte los principios éticos antes mencionados, por otra el miedo a verse envuelto en un proceso judicial derivado de la sospecha de una mala praxis, una de las experiencias más traumáticas por las que puede pasar el profesional sanitario, incluso cuando este se resuelve favorablemente ${ }^{17}$.

Desde el inicio de los SNI como fuente de información para conocer los problemas de seguridad del paciente se detectaron los primeros problemas. Los profesionales no declaraban los EA por una falta de confianza en la utilidad del sistema y también por miedo a las consecuencias legales ${ }^{18,19}$.

Para facilitar la utilización de los sistemas de notificación voluntarios el Institute of Medicine (IOM) en su informe "To err is Human" propone SNI no punitivos, lo que puede redundar en la necesidad de un cambio legislativo en aras de proteger al declarante ${ }^{20}$. Resulta interesante observar cómo en países como el Reino Unido con la Agencia de Seguridad del Paciente (NPSA), Dinamarca (The Danish Society for Patient Safety), Suecia, EEUU (National Center for Patient Safety, NCPS), Australia... con amplia experiencia en el desarrollo de estos sistemas, se señala ya la necesidad de no utilizar la información obtenida de estos sistemas con fines sancionadores o culpabilizadores. De este modo desarrollan registros donde se garantiza la confidencialidad, el carácter anónimo de los datos, su empleo únicamente para el aprendizaje y prevención, voluntariedad... pero sobretodo y además, crean un marco legal adecuado, donde se excluye cualquier consecuencia de carácter disciplinario, laboral o penal para el notificante por el mero acto de la notificación, que es lo que realmente posibilita su amplio desarrollo y difusión ${ }^{21,24}$. 
Durante los últimos años en nuestro país han proliferado los sistemas de notificación de incidentes. Se han propuesto a los profesionales sanitarios como una eficaz herramienta de aprendizaje y de mejora. Sin embargo desconocemos los conocimientos que poseen dichos profesionales sobre los problemas legales que pueden derivarse del uso de estos sistemas de notificación.

\section{Método}

Estudio descriptivo transversal en el que se utilizó un cuestionario elaborado "ad hoc" para averiguar los conocimientos y actitudes de los profesionales sanitarios ante los problemas éticos y legales de los sistemas de notificación.

La población del estudio se encuentra constituida por profesionales sanitarios del Servicio Aragonés de Salud (SALUD) que desarrollan su labor tanto en atención primaria como en especializada; médicos, personal de enfermería y profesionales en puestos directivos y de gestión.

El cuestionario se difundió en papel mediante muestreo por conveniencia y en bola de nieve.

Cuestionario (Anexo 1): la elaboración del cuestionario se basó en el modelo teórico de la responsabilidad jurídica derivada de la praxis de las profesiones médico sanitarias ${ }^{25}$. El cuestionario incluye cinco ítems que profundizan sobre algunos de los problemas legales más debatidos de los SNI y dos ítems sobre el derecho de los pacientes a recibir información sobre los problemas relacionados con la seguridad. Se plantea una reflexión final, informando a los respondedores sobre la falta de protección de los datos notificados ante un requerimiento legal.

La encuesta recoge información sobre el ámbito en el que se desarrolla la actividad profesional, la categoría profesional y la formación previa en SP.

Para facilitar y simplificar la presentación de las respuestas, se han recodificado las variables que permitían responder en cinco o más categorías, en tres categorías. Una vez recodificadas, se han calculado las frecuencias relativas de cada una de las categorías. El análisis estadístico de las respuestas se realizó con el programa estadístico SPSS. Se calculó la frecuencia de respuesta y se utilizó la prueba de Chi cuadrado para el contraste de proporciones. 


\section{Resultados}

Un total de 150 cuestionarios fueron distribuidos, obteniéndose una respuesta de 123 personas (tasa de respuesta del 82\%). La descripción de la población puede verse en la tabla 1:

\begin{tabular}{l|lcc}
\hline Características & Categorías & N & $\%$ \\
\hline \multirow{2}{*}{ Sexo } & Varón & 44 & 35,8 \\
& Mujer & 79 & 64,2 \\
Ámbito laboral & Atención Primaria & 46 & 37,4 \\
& Atención especializada & 51 & 41,5 \\
& Otros & 26 & 21,1 \\
Categoría profesional & Médico & 77 & 62,6 \\
& Enfermera & 37 & 30,1 \\
\multirow{5}{*}{ Relación directa con el paciente } & Otro & 9 & 7,3 \\
& & & \\
\multirow{3}{*}{ Formación en SP } & No & 94 & 76,4 \\
& & 29 & 23,6 \\
& No & 48 & 39 \\
& Básica & 62 & 50,4 \\
& Avanzada & 13 & 10,6
\end{tabular}

Tabla 1: Características de la población.

En las tablas 2, 3 y 4 se detallan los resultados globales de la encuesta.

\begin{tabular}{|c|c|c|c|}
\hline Ítem & Respuestas & $\mathbf{N}$ & $\%$ \\
\hline $\begin{array}{l}\text { 1.1.- Todos los incidentes con consecuencias graves } \\
\text { para el paciente deben ser investigados para reparar el } \\
\text { daño causado al paciente y su familia, aunque esto } \\
\text { pueda suponer una sanción penal o administrativa }\end{array}$ & $\begin{array}{l}\text { De acuerdo } \\
\text { En desacuerdo } \\
\text { Sin opinión definida }\end{array}$ & $\begin{array}{l}4 \\
4 \\
5\end{array}$ & $\begin{array}{l}6,4 \\
1,4 \\
2,2\end{array}$ \\
\hline $\begin{array}{l}\text { 1.2.- El paciente tiene derecho a conocer los } \\
\text { incidentes notificados por los servicios sanitarios a los } \\
\text { que él confía su salud }\end{array}$ & $\begin{array}{l}\text { De acuerdo } \\
\text { En desacuerdo } \\
\text { Sin opinión definida }\end{array}$ & $\begin{array}{l}8 \\
0 \\
5\end{array}$ & $\begin{array}{c}7 \\
2,5 \\
0\end{array}$ \\
\hline $\begin{array}{l}\text { 1.3.- El paciente tiene derecho a conocer aquellos } \\
\text { incidentes notificados que le atañen directamente }\end{array}$ & $\begin{array}{l}\text { De acuerdo } \\
\text { En desacuerdo } \\
\text { Sin opinión definida }\end{array}$ & $\begin{array}{l}94 \\
14 \\
15\end{array}$ & $\begin{array}{l}76,4 \\
11,4 \\
76,4\end{array}$ \\
\hline
\end{tabular}

Tabla 2: Opinión de los profesionales sobre los derechos de los pacientes en lo que respecta a los SNI. 


\begin{tabular}{|c|c|c|c|}
\hline Ítem & Respuestas & $\mathrm{N}$ & $\%$ \\
\hline $\begin{array}{l}\text { 2.1. ¿Cree usted que notificar incidentes y efectos } \\
\text { adversos graves con fines de aprender sobre ellos, } \\
\text { analizar las circunstancias en que se produjeron y } \\
\text { evitar que se repitan puede tener como contrapartida } \\
\text { problemas legales? }\end{array}$ & $\begin{array}{l}\text { Sí } \\
\text { No } \\
\text { NS/NC }\end{array}$ & $\begin{array}{l}81 \\
26 \\
16\end{array}$ & $\begin{array}{c}65,9 \\
21,1 \\
13\end{array}$ \\
\hline $\begin{array}{l}\text { 2.2. ¿Cree usted que la legislación española protege al } \\
\text { que declara un incidente o un EA a través de un } \\
\text { sistema de registro? }\end{array}$ & $\begin{array}{l}\text { Sí } \\
\text { No } \\
\text { NS/NC }\end{array}$ & $\begin{array}{l}19 \\
43 \\
61\end{array}$ & $\begin{array}{c}15,4 \\
35 \\
49,6\end{array}$ \\
\hline $\begin{array}{l}\text { 2.3. ¿Cree usted que un sistema de registro puede } \\
\text { llegar a ser utilizado como fuente de datos en un } \\
\text { proceso judicial? }\end{array}$ & $\begin{array}{l}\text { Sí } \\
\text { No } \\
\text { NS/NC }\end{array}$ & $\begin{array}{l}76 \\
15 \\
32\end{array}$ & $\begin{array}{l}61,8 \\
12,2 \\
26\end{array}$ \\
\hline $\begin{array}{l}\text { ¿Cree usted que cabe la posibilidad de que un sistema } \\
\text { de registro sea utilizado como un sistema de delación? }\end{array}$ & $\begin{array}{l}\text { Sí } \\
\text { No } \\
\text { NS/NC }\end{array}$ & $\begin{array}{l}52 \\
20 \\
51\end{array}$ & $\begin{array}{l}42,3 \\
16,3 \\
51\end{array}$ \\
\hline
\end{tabular}

Tabla 3: Opinión de los profesionales sobre los problemas legales de los SIN.

\begin{tabular}{lcc}
\hline Respuesta & N & $\%$ \\
\hline $\begin{array}{l}\text { Con independencia de lo que acabo de leer, notificaría los incidentes de } \\
\text { los que yo fuera conocedor, tanto si han provocado daño como si no }\end{array}$ & 15 & 12,2 \\
$\begin{array}{l}\text { Sólo notificaría los incidentes que no hubieran llegado a provocar daño } \\
\text { al paciente }\end{array}$ & 3 & 2,4 \\
No notificaría ningún tipo de incidente & 16 & 13,0 \\
$\begin{array}{l}\text { Notificaría los incidentes de los que fuera conocedor, teniendo cuidado } \\
\text { de respetar el anonimato de los profesionales implicados en el suceso }\end{array}$ & 86 & 69,9 \\
No contesta & 3 & 2,4 \\
\hline
\end{tabular}

Tabla 4: respuesta de los profesionales a la reflexión final propuesta.

El $47,2 \%$ de los participantes considera que el paciente tiene derecho a conocer la seguridad del Sistema Sanitario donde es atendido, y un $76,4 \%$ opina que el paciente tiene derecho a conocer si ha existido algún EA durante su atención. Se evidencia una diferencia estadísticamente significativa entre los profesionales asistenciales y aquellos que no atienden pacientes directamente, así como entre los médicos y el personal de enfermería.

El 77\% de los encuestados está de acuerdo con investigar los incidentes, incluso cuando de esta investigación pueda derivarse una sanción penal o administrativa. Se observa diferencia estadística entre médicos y enfermeras (el 100\% de los profesionales de enfermería están a favor de investigar frente al $68,8 \%$ de los médicos) y entre profesionales asistenciales y los que no lo son, estando de acuerdo con investigar el $73 \%$ de los profesionales asistenciales y el $93 \%$ de los que no atienden pacientes directamente. 
El $67,4 \%$ de los encuestados juzga que se pueden derivar consecuencias legales de la notificación de los incidentes, sin embargo el $50 \%$ desconoce si el declarar confiere o no protección al declarante. El 63\% considera que la información que contienen los SNI puede ser empleada en un juicio, y hasta un $25 \%$ ignora si puede o no ser empleada para tal fin. Por otra parte, un $42,4 \%$ de profesionales considera que este tipo de sistemas puede constituir un modo de delación. En este apartado no existen diferencias significativas entre los grupos de encuestados.

Una vez informados de la posibilidad de que la notificación derive en problemas legales, el $70 \%$ responde que notificaría, si bien poniendo especial atención en respetar el anonimato de los profesionales implicados en el suceso.

\section{Conclusiones}

Es probable que el tamaño muestral del estudio sea pequeño pero se ha conseguido que se encuentren representadas las diferentes realidades de nuestro sistema sanitario: atención especializada y primaria, médicos y personal de enfermería, profesionales en contacto directo con los pacientes y profesionales cuya labor no es asistencial.

Por otra parte, somos conscientes de que no se ha podido aplicar ningún método que mida la estabilidad, es decir no se ha podido realizar la prueba del test-retest en la misma población, puesto que solamente se ha realizado una única medición en el tiempo a través del cuestionario.

Además, como no existe ningún instrumento validado para valorar la percepción que pretendemos evaluar (pues no existe ninguna otra encuesta de referencia), no hemos podido comparar nuestros resultados con los de otros estudios.

El primer problema planteado por la encuesta, la dualidad entre la necesidad de investigar para reparar el daño y la posibilidad de que de esta investigación se deriven problemas legales se resuelve a favor del paciente, con una mayoría de profesionales dispuestos a asumir el riesgo. Sin embargo hay que señalar que son los médicos asistenciales quienes se muestran más reticentes a esta investigación. Es posible que los profesionales de enfermería y aquellos que no atienden pacientes directamente no sientan la responsabilidad legal tan directamente sobre ellos como los médicos asistenciales.

Respecto al derecho del paciente a conocer la seguridad del sistema que le atiende y los problemas de seguridad que le afectan personalmente, el personal de enfermería considera que el paciente tiene derecho a ser conocedor de los EA derivados de su asistencia sanitaria, no así los no asistenciales quienes abogaban por un SNI en el que los profesionales debían declarar, aunque el sistema no estuviera exento de carácter sancionador. Quizás la percepción de la responsabilidad que tiene sistema en la génesis de los EA, sea la responsable del cambio de opinión en la respuesta de este item.

En el momento actual una de las mayores preocupaciones de las administraciones de diversos países, es la posibilidad de establecer una política de transparencia en la comunicación de los 


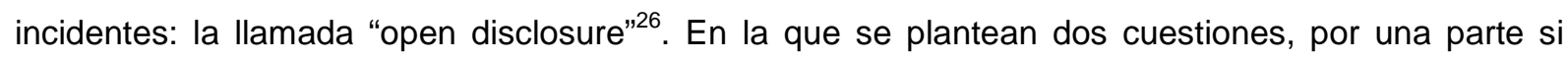
deben hacerse públicos los incidentes que los sistemas de salud miden habitualmente (infecciones asociadas a la asistencia sanitaria, caídas, eventos centinela...), y por otra la necesidad de comunicar a los pacientes de un modo abierto y honesto los incidentes ocurridos en el proceso de su atención sanitaria. Este respecto confronta el derecho del paciente con la ausencia de una legislación específica que proteja a ambos.

El aspecto más preocupante de las preguntas derivadas de las implicaciones legales de los SNI es el gran desconocimiento con el que cuentan los profesionales. Las cuatro preguntas presentan un elevado porcentaje (hasta el 50\%) de indefinición, siendo lo más llamativo que no existe diferencia alguna entre los profesionales que afirman haber recibido información previa sobre los problemas legales que ocasionan los SNI y los que no la han recibido. Sin embargo esta diferente percepción quizás sólo traduzca las posibles colisiones normativas presentes en el marco jurídico español, pudiendo aparecer enfrentados el deber de denuncia y el deber de secreto profesional ${ }^{27-29}$.

Pero al informar sobre la posibilidad de afrontar problemas legales, una mayoría de los encuestados insisten en la necesidad de declarar, si bien protegiendo el anonimato de los implicados. Esta respuesta traduce la favorable predisposición de los profesionales sanitarios ante los SNI y deja entrever que, si fuera posible garantizar la seguridad jurídica del declarante, los SNI se convertirán en un arma eficaz para mejorar la seguridad del paciente.

\section{Agradecimientos}

Al Dr. Carlos Aibar Remón y a la Dra. Teresa Gimenez Julvez, por su apoyo y asesoramiento en la elaboración de este trabajo. A los profesores del Master de Bioética y Derecho de la Universidad de Barcelona, por proporcionarnos los impagables conocimientos necesarios para desarrollar este proyecto. 


\section{Bibliografía}

- Aibar C., Aranaz J. Seguridad del paciente y prevención de eventos adversos relacionados con la asistencia sanitaria. MSC. http://www.seguridaddelpaciente.es/formacion/tutoriales/MSCCD1/contenidos/. Última entrada: 10/abril/2013

- LEY 41/2002, de 14 de noviembre, básica reguladora de la autonomía del paciente y de derechos y obligaciones en materia de información y documentación clínica. BOE 274 de 15 de noviembre de 2002: 40.126-302.

- Pellegrino ED Toward a reconstruction of medical morality.J Med Humanit Bioeth. 1987 SpringSummer; 8(1):7-18.

- Martín Delgado MC, Cabré Pericas, L. Aspectos éticos y legales sobre la seguridad del paciente. Revista de Bioética y Derecho. 2009. 15: 6-14.

- Pichert J, Hickson G. Communicating risk to patients and families. En Vincent C (ed). Clinical risk management: enhancing patient safety 2nd ed. London: BMJ Publications, 2001.

- Un proyecto de la Fundación ABIN, la Fundación ACP-ASIM y la Federación Europea de Medicina Interna. La profesión médica en el nuevo milenio: estatutos para la regulación de la práctica médica. Med Clínica 2002; 118 (18): 704-706.

- Itoh K, Andersen HB, Madsen MD, Østergaard D, Ikeno M. Patient views of adverse events: comparisons of self-reported healthcare staff attitudes with disclosure of accident information. Appl Ergon. 2006;37 (4):513-23.

- Aranaz-Andrés JM, Aibar-Remón C, Vitaller-Murillo J, Ruiz-López P, Limón-Ramírez R, TerolGarcía E, the ENEAS work group. Incidence of adverse events related to health care in Spain: Results of the Spanish National Study of Adverse Events. J Epidemiol Community Health. 2008; 62:1022-9

- Sharpe VA. Promoting patient safety. An ethical basis for policy deliberation. Hastings Cent Rep. 2003 Sep-Oct; 33(5):S3-18

- Simón Lorda P. Ética y seguridad de los pacientes. Humanitas, Humanidades Médicas. 2005;8:145-60.

- Revisión Bibliográfica sobre Trabajos de Costes de la "No Seguridad del Paciente". Madrid: Ministerio de Sanidad y Consumo; 2008. En URL: http://www.msc.es/organizacion/sns/planCalidadSNS/docs/CostesNoSeguridadPacientes.pdf. Última entrada 10 de abril de 2013.

- OMS. Seguridad del paciente. En URL: http://www.who.int/patientsafety/es/. Última entrada 10 de abril de 2013. 
- Plan de Calidad del SNS. En URL: http://www.msc.es/organizacion/sns/planCalidadSNS lec pnc03.htm

- Scott SD, Hirschinger LECox KR et al. The nature history of recovery for the health care provider "second victim" after adeverse patient events. Qual Saf Health Care 2009; 18: 325-330.

- Schwappach DL, Boluarte TA. The emotional impact of medical error involvement on physicians: a call for leadership and organizational accountability. Swiss med Wkly 2009; 139: 9-15.

- When the things go wrong. En URL: http://www.macoalition.org/documents/respondingToAdverse Events.pdf).

- Joint Comission Perspectives. Vol 30. 2010, Issue 8

- Vincent C, Stanhope N, Crowley-Murphy M.Reasons for not reporting adverse incidents: an empirical study.J Eval Clin Pract. 1999 Feb;5(1):13-21.

- Leape LL. Why should we report adverse incidents? J Eval Clin Pract. 1999 Feb;5(1):1-4.)

- Kohn LT, Corrigan JM, Donaldson MS, eds. To err is human: building a safer health system. Washington, D.C.: National Academy Press, 2000.

- Agencia Nacional para la Seguridad del Paciente (NPSA)- Sistema Nacional de Salud, Reino Unido, La seguridad del paciente en siete pasos. Agencia de Calidad, Ministerio de Sanidad y Consumo, Madrid, 2006.

- Act on Patient Safety in the Danish Health Care System. Act no. 429 of June 10, 2003. Available at www.patientsikkerhed.dk/About.

- Patient safety and quality improvement act of 2005 (PublicLaw109-41)

- Health Insurance (Quality Assurance Confidentiality) Amendment Act. 201. 1992.

- Establecimiento de un sistema nacional de notificación y registro de incidentes y eventos adversos en el sector sanitario: aspectos legales. Madrid: Ministerio de Sanidad y Política social; 2009.

- Aibar C., Aranaz J. Seguridad del paciente y prevención de eventos adversos relacionados con la asistencia sanitaria. MSC. Unidad 9: La comunicación del riesgo: compartiendo decisiones con los pacientes. En URL: http://www.seguridaddelpaciente.es/formacion/tutoriales/MSC-CD1/contenidos/ unidad9.4.html. Última entrada 10 de abril de 2013.

- Art 142 y 152 Código Penal.

- Art. 24.2 de La Constitución Española

- Romeo Casabona CM, Urruela Mora A. El establecimiento de un sistema nacional de notificación y registro de eventos adversos en el sector sanitario aspectos legales. Capítulo 1: Tensiones y conflictos de Lege Lata en la configuración de un sistema nacional de notificación y registro de eventos adversos. Pág 1-74. Granada Comares 2010. 


\section{Anexo 1}

Cuestionario sobre la percepción de los problemas legales de los SIN. Este cuestionario es parte del cuestionario PELSN: PERCEPCIÓN DE LOS PROBLEMAS ÉTICOS Y LEGALES DE LOS SISTEMAS DE NOTIFICACIÓN DE INCIDENTES RELACIONADOS CON LA SEGURIDAD DEL PACIENTE

\section{Sección 1}

Se utilizará la siguiente escala a lo largo de esta sección:

\begin{tabular}{|l|l|l|l|l|}
\hline $\begin{array}{l}\text { 1: totalmente en } \\
\text { desacuerdo }\end{array}$ & 2: en desacuerdo & $\begin{array}{l}\text { 3: ni acuerdo ni } \\
\text { desacuerdo }\end{array}$ & 4: de acuerdo & $\begin{array}{l}\text { 5: totalmente de } \\
\text { acuerdo }\end{array}$ \\
\hline
\end{tabular}

\begin{tabular}{|l|l|l|l|l|l|}
\hline & 1 & 2 & 3 & 4 & 5 \\
\hline $\begin{array}{l}\text { 1.1. Todos los incidentes con consecuencias graves para el paciente } \\
\text { deben ser investigados para reparar el daño causado al paciente y su } \\
\text { familia, aunque esto pueda suponer una sanción penal o administrativa }\end{array}$ & & & & & \\
\hline $\begin{array}{l}\text { 1.2. El paciente tiene derecho a conocer los incidentes notificados por } \\
\text { los servicios sanitarios a los que él confía su salud }\end{array}$ & & & & \\
\hline $\begin{array}{l}\text { 1.3. El paciente tiene derecho a conocer aquellos incidentes } \\
\text { notificados que le atañen directamente }\end{array}$ & & & & \\
\hline
\end{tabular}

\section{Sección 2}

Se responderá: sí, no o no sabe/no contesta (NS/NS)

\begin{tabular}{|l|l|l|l|}
\hline & Sí & No & NS/NC \\
\hline $\begin{array}{l}\text { 2.1. ¿Cree Vd que notificar incidentes y efectos adversos graves con } \\
\text { fines de aprender sobre ellos, analizar las circunstancias en que se } \\
\text { produjeron y evitar que se repitan puede tener como contrapartida } \\
\text { problemas legales? }\end{array}$ & & & \\
\hline $\begin{array}{l}\text { 2.2. ¿Cree usted que la legislación española protege al que declara } \\
\text { un incidente o un EA a través de un sistema de registro? }\end{array}$ & & & \\
\hline $\begin{array}{l}\text { 2.3. ¿Cree usted que un sistema de registro puede llegar a ser } \\
\text { utilizado como fuente de datos en un proceso judicial? }\end{array}$ & & & \\
\hline $\begin{array}{l}\text { ¿Cree usted que cabe la posibilidad de que un sistema de registro } \\
\text { sea utilizado como un sistema de delación? }\end{array}$ & & & \\
\hline
\end{tabular}




\section{Reflexión final}

Lea atentamente la siguiente frase:

Los datos conocidos en el marco de la configuración de un sistema de notificación de efectos adversos, tanto por parte del gestor de la base de datos en la que se recopilaran los mismos, como por parte de comisiones técnicas encargadas del estudio de casos, en ningún caso gozarían de protección alguna frente al requerimiento judicial, particularmente en materia penal. (Texto modificado de: Romeo Casabona CM, Urruela Mora A, Libano Beristain A. Establecimiento de un sistema nacional de notificación y registro de incidentes y eventos adversos: aspectos legales. La notificación de eventos adversos en el sector sanitario: perspectiva de derecho comparado: segundo informe, diciembre 2007. Madrid: Ministerio de Sanidad y Consumo; 2008.)

En relación al mismo señale con cuál de las siguientes afirmaciones se siente más identificado:

1. Con independencia de lo que acabo de leer, notificaría los incidentes de los que yo fuera conocedor, tanto si han provocado daño como si no.

2. Sólo notificaría los incidentes que no hubieran llegado a provocar daño al paciente

3. No notificaría ningún tipo de incidente.

4. Notificaría los incidentes de los que fuera conocedor, teniendo cuidado de respetar el anonimato de los profesionales implicados en el suceso.

\section{Información complementaria}

Esta información ayudará al análisis de los resultados de la evaluación

1. Sexo: V/M

2. Categoría profesional: Médico/a / Enfermero/a / Otros

3. En su puesto de trabajo mantiene habitualmente un contacto directo con los pacientes: Sí / No

4. Ámbito de trabajo: Atención primaria / Especializada / Otros

5. Tiene formación específica en Seguridad del Paciente:

No

Básica: asistencia a cursos $<30 \mathrm{~h}$

Avanzada: experto universitario, master... 